\title{
Risk Assessment of Bisphenol A (BPA) in Thermochromic Textiles
}

\author{
S. S. HE ${ }^{1, a}$, M. Y. WEI ${ }^{2, b}$, F. LIU ${ }^{2, c}$, W. L. XUE ${ }^{1,3, d^{*}}$, L. D. CHENG $^{1, e}$ \\ ${ }^{1}$ Key Lab of Eco-Textile, Ministry of Education, Donghua University, Shanghai 201620, People's \\ Republic of China
}

${ }^{2}$ Shanghai Entry-Exit Inspection and Quarantine Bureau, Shanghai 200135, People's Republic of China

${ }^{3}$ Foshan nanhai Southern Technology Innovation Center co., LTD

*Corresponding author: xwl@dhu.edu.cn

Keywords: Functional textiles, Thermal-sensitive textiles, Bisphenol A (BPA), Risk assessment.

\begin{abstract}
Bisphenol A (short as BPA) is usually used for thermochromic products, and in recent years, it begins to be applied in functional textile with thermochromic performance. As a kind of environmental hormone, BPA is generally considered as chemicals with risks for its embryo toxicity and teratogenicity, which can result in cancer. In this study, thermochromic textiles in current market are collected for samples as many as 12 pieces. Experiments have been done for the determination of BPA in these samples to work out the health risks of thermochromic textiles. The results request laws or regulations on content of BPA in functional textile, because the HPLCFLD/DAD test results showed detection of BPA in all samples, and some of which even have a high BPA content which will lead high risk to consumers and environment
\end{abstract}

\section{Introduction}

Bisphenol A, short as BPA, is a kind of environmental hormone, which has been testified as cancerogen with embryo toxicity and teratogenicity. Since 2008, in countries and regions like Sweden, Norway, Canada, America, EU and China, BPA has been banned in application of baby bottles and food wrappers. For textile industry, the most common usage of BPA is to work as thermochromic chromogenic reagent in print. When in touch with human, BPA in textiles can be released and lead to health problems, such as reproductive system problems and effect on cellular development ${ }^{[1]}$. There have been various functional textiles with different performances, and for colour-changing ones, BPA is a common used thermochromic dye in textile industry ${ }^{[2]}$. Therefore, thermal-sensitive textiles in current market were collected and tested in this research by highperformance liquid chromatography with fluorescence detection / diode array detection (HPLCFLD/DAD), to figure out their BPA content. Based on the experimental data, this study can make predict or early warnings for functional textiles with thermal-sensitive performance so that the market can make prevention and control as early as possible ${ }^{[3]}$.

By far, the most widely used chromotropic dye is thermo-sensitive dye, and it is the kind of dye in best control in process setup. Thermochromic dyes will change its color when temperature turns up, and recover when the temperature turns down. Thermochromic dyes are usually classified into three categories, which are inorganic dyes, dyes doped with liquid crystals and organic ones, based on their colour-change mechanism ${ }^{[4]}$. Among them, inorganic dyes are usually restricted by its toxicity, while the last ones are only applied in limited application for its high cost. In textile industry, organic thermochromic agents are usually used ${ }^{[5-6]}$. Compounded with different materials, they conclude the following constituents ${ }^{[7]}$ :

(1) Electron donor, which decides the hue. The most typical one is the agents belonging to triarylmethane, such as crystal violet lactone;

(2) Electron acceptor, working as the chromogenic reagent, leads to different shades. These kinds of agents are usually classified as phenols and new non-polyphenols.

(3) Materials working as solvent, such as fatty acid and higher aliphatic alcohol.

(4) Other addictive ${ }^{[8]}$. 


\section{Experimental Procedure:}

\section{Retail Samples}

In this research, 12 color-changing textiles were purchased from in retail outlets in Chinese market, including bath towels, hand towels or infant towels with color-changing patterns. Samples in experiments are taking from the thermochromic parts of the textiles, without separating the printing and base cloth.

\section{Reagent and Instruments}

Chemicals and solvents: BPA reference standards and methyl alcohol (HPLC grade) both from ANPEL (China). Ultrasonic cleaners (Elma P180H), liquid chromatograph (Agilent 1260), reactor, PTFE syringe-driven filter.

\section{Sample Preparation}

$0.5 \mathrm{~g}$ thermochromic parts of the textiles were weighed and cut out into powder. The powder were put in reactor and $20 \mathrm{~mL}$ methyl alcohol as solvent were accurately added. Shake the reactor in order to allow all sample powder immersed into the solvent. The BPA in sample powder was extracted by ultrasonic for 30min.

Extraction was filtrated through the PTFE syringe-driven filter for high-performance liquid chromatography with fluorescence detection / diode array detection (HPLC-FLD/DAD) preparation.

\section{Preparation of Standard Solution}

BPA was dissolved into methyl alcohol for the preparation of BPA reference solution, in which the concentration of BPA was $100 \mu \mathrm{g} / \mathrm{mL}$. Dilute the BPA reference solution with methyl alcohol for different concentrations of compliance with standard work curve.<smiles>CC(C)(c1ccc(O)cc1)c1ccc(O)cc1</smiles>

Figure 1 The structure of bisphenol A.

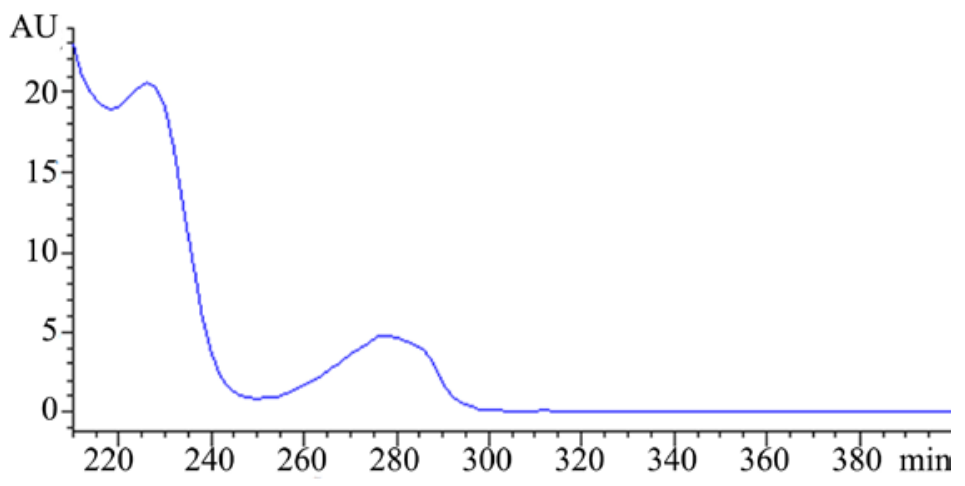

Figure 2 The ultraviolet spectra of bisphenol A. 


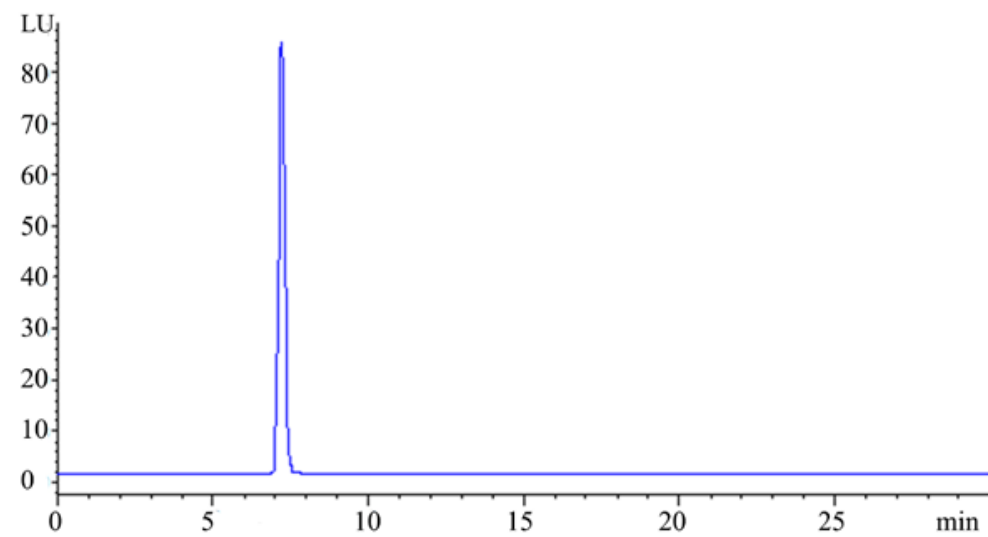

Figure 3 The FLD spectra of bisphenol A.

\section{Measurement}

Diphenol propane, known as BPA, has a structural formula shown in Figure 1. The conjugated double bonds in its benzene ring presents a very strong absorbance in ultraviolet detector. When the excitation wavelength and emission wavelength are set at $275 \mathrm{~nm}$ and $313 \mathrm{~nm}$, separately, it can show a strong fluorescence signal in BPA test (Figure 2\&3). Thus, it can be reasonable to analysis the content of BPA with HPLC-FLD for qualitative analysis while HPLC-FLD for quantitate analysis.

\section{Method}

The injection volume was $10 \mu \mathrm{L}$. The $\mathrm{C} 18$ chromatographic column $(250 \mathrm{~mm} \times 4.6 \mathrm{~mm} \times 5 \mu \mathrm{m})$ was selected, the mobile phase was composed of methyl alcohol-water (65:35), the flow rate was $1.0 \mathrm{~mL} / \mathrm{min}$. The detection wavelength was $280 \mathrm{~nm}$, and the spectral canning range is from $200 \mathrm{~nm}$ to $400 \mathrm{~nm}$.

\section{Results and Discussion}

\section{Results}

12 brands of thermochromic textiles purchased and analysed with the test method mentioned above indicated that all thermochromic parts of the samples were made of BPA. Table 1 shows the BPA content of the samples. Of the whole 12 samples, the concentrations of BPA rang from $111 \mathrm{mg} / \mathrm{kg}$ to $1426 \mathrm{mg} / \mathrm{kg}$, and most of the concentration distributed around $600 \mathrm{mg} / \mathrm{kg}$ and $1000 \mathrm{mg} / \mathrm{kg}$.

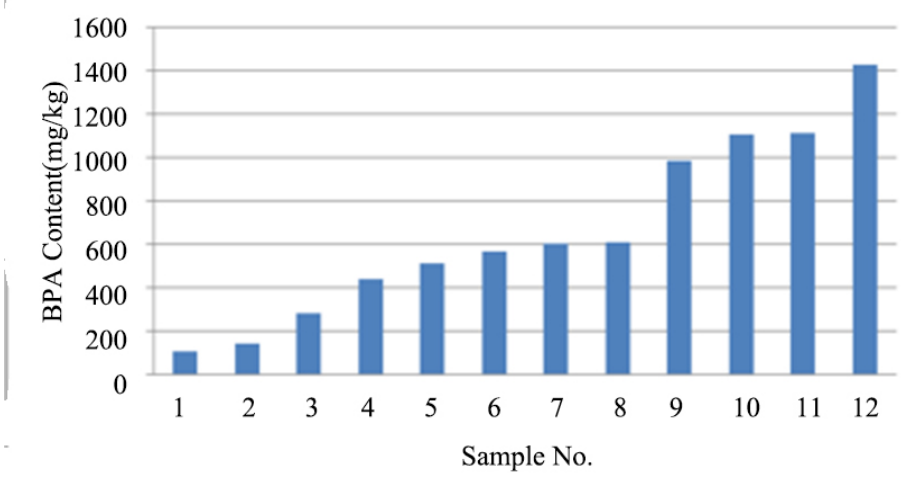

Figur 4 Bisphenol A content distribution of samples. 
Table 1 Result of bisphenol A testing.

\begin{tabular}{l|l}
\hline Sample No. & BPA Content(mg/kg) \\
\hline 1 & 111 \\
2 & 143 \\
3 & 282 \\
4 & 441 \\
5 & 514 \\
6 & 567 \\
7 & 606 \\
8 & 608 \\
9 & 982 \\
10 & 1108 \\
11 & 1112 \\
12 & 1426 \\
\hline
\end{tabular}

As color developing agent in thermochomic dye, BPA has an influence on the shades of textile colour by its quantity. With the increase of BPA content, it has more chances to bind to dyes, therefore, colour of textiles gets darker. However, when the content of BPA increases to a certain level of concentration, the solvent becomes saturated with dyes or BPA, thus the redundant BPA will not combined with dyes any more, which means, BPA cannot affect the shade of textiles beyond a certain concentration.

\section{Risk Analysis}

BPA has the effect of natural hormone or inhibition of natural hormone, which can interfere with the regulation of normal function in nerve system, immune system and endocrine system, and therefore pose a risk to human health and our environment ${ }^{[9]}$.

The use of BPA has drawn attention of countries all around the world. In October 2008, Canada classified BPA as one of the poisonous chemicals. In 2009, American released ban on BPA on its use in "reusable food container" and "other food containers". On May 1, 2011, law named as 2011/10/EU took effect in European Union provides that the limit value of BPA migrating in food content plastic is $0.6 \mathrm{mg} / \mathrm{kg}^{[10]}$. In China, there are national standard GB 13116-1991<Hygienic standard for polycarbon resin used as food containers and packging materials>, and GB14942$1994<$ Hygienic standard for polycarbonate products used as food containers and packaging materials $>$, which provide that the maximum migration value of phenol in resin materials and products should be no more than $0.05 \mathrm{mg} / \mathrm{kg}$. National standard GB 9685-2008< Hygienic standards for uses of additives in food containers and packaging materials $>$ sets the limit value for BPA migration in adhesive and coating should be $0.6 \mathrm{mg} / \mathrm{kg}{ }^{[11]}$. There has been no limit requirement for BPA in textile by far in the world.

Bath towel or hand towel contact with human skin closely in use. If there is BPA contained in thermochomic dye, it may lead danger to human or environment. Relative high detectable BPA content in all textiles in this analysis means that BPA can pose a serious risk to human and environment. So there should be more and more investment on prevent or restrictions of BPA's usage, especially, in textile industry, and build up an effective and efficient risk assessment mechanism, in order to ensure the ban or restrictions on BPA application in infant textile products and textiles with direct contact with skin.

\section{Conclusion}

In this study, the 12 thermochomic functional textiles all present a relative high content of BPA, which means a high risk resulted by BPA. Therefore the study provides warnings on BPA risk in 
textile use which has never been noticed before, and also suggestions on the establishment of risk assessment of BPA to regulate its limit value in product, prevent and restrict its application, and also provide a reliable basis for market supervision.

\section{Acknowledgement}

This work was financially supported by the Chinese Fundamental Research Funds for the Central Universities (15D110173) and Scientific Research Project of AQSIQ PRC (2015IK238).

\section{References}

[1]Hu, J.-Y., Aizawa, T., Ookubo, S., Products of aqueous chlorination of bisphenol A and their estrogenic activity. Environ. Sci. Technol. 36, pp. 1980-1987, 2002.

[2]Textor, T., Schröter, F., and Schollmeyer, E.: 'Functionalisation of textiles with nanotechnology'. In MRS Proceedings. Cambridge University Press, January 2006, Paper 0920-S01

[3]Mountfort, K.A., Kelly, J., Jickells, S. M. \& Castle, L. Investigations into the potential degradation of polycarbonate baby bottles during sterilization with consequent release of bisphenol A. Food Additives \& Contaminants, 14(6-7), pp. 737-740. 1997.

[4]Youchang Liu, Yan Bu, Lu Xue, New functional materials--Application of coloring materials to textiles. Chinese Fiber Inspection, (19), pp. 77-79, 2011.

[5]Zhen Wan, Wei Wang, Jun Xie, Thermal-sensitive color-changing material and its application in textiles, Journal of Silk in Chinese, (8), pp. 44-46, 2003.

[6]TANG, Z. R., ZHANG, X., Reversible Organic Thermochromic Materials [J]. Journal of East China Geological Institute, (1), pp. 50-54, 2002.

[7]Aitken,Aitken, D., Burkinshaw, S. M., Griffiths, J. and Towns, A. D. (1996), Textile applications of thennochromic systems. Review of Progress in Coloration and Related Topics, 26: 1-8. doi: 10.1111/j.1478-4408.1996.tb00105.x

[8]Weijin Liu, Tai Liang, Suzhi Li, Preparation and properties of reversible thermochromism materials, Journal of Silk in Chinese, (5), 31-33, 2008.

[9]Yonggang Gao, Yanyan Zhang, Jianguo Gao, Determination of bisphenol A from toys and food contact materials by derivatization and gas chromatography-mass spectrometry, Chinese journal of Chromatography, 30(10), 1017-1020, 2012.

[10]Staples, C.A., Dorn, P.B., Klecka, G.M., O’Block, S.T., Harris, L.R., A review of the environmental fate, effects, and exposures of bisphenol A. Chemosphere, 36, pp. 2149-2173, 1998.

[11]Yongmei Guo, Analysis of health hazards, legal restrictions and regulations for BPA, Modern Food Science and Technology in Chinese, (5), pp. 549-551, 2012. 